\title{
Spin, gravity, confinement
}

\author{
Ivanhoe Pestov* \\ JINR \\ E-mail:pestovetheor.jinr.ru
}

It is supposed that the physical space has extra dimension. Possible effects of this innovation are investigated on the example of the simplest mathematical space - the four dimensional Euclidian space. Symmetry properties and geometrical constructions inherent in this space only are established. It is shown that in the space in question there are two natural frames of orthogonal vector fields of equal length, represent its internal property. On this ground, we establish the existence of a coherent system of thirteen points that constitute a rigid structure in the form of two pairs of regular tetrahedrons with a common vertex. When this vertex moves along the given trajectory, the tetrahedrons of each pair are in the relative rotation and all tetrahedrons in general can also pulse. Thus, it is demonstrated that the origin and the nature of a rotational motion are clearly visible only in four dimensions. A pair of Dirac equations associated with the natural frames on the four-dimensional Euclidian space is considered. Comparing these equations with the original Dirac equation in the Minkowski space-time we show that there are two space-time structure on the four-dimensional Euclidian space. With this, the rational proof of the existence of leptons and quarks, lepton-quark symmetry and confinement is obtained.

XXII International Baldin Seminar on High Energy Physics Problems

15-20 September, 2014

JINR,Dubna,Russia

${ }^{*}$ Speaker. 


\section{Introduction}

One of the primary goals of physics is to understand the variety of physical reality in a unified way: a single mathematical framework in which all fundamental forces and units of matter can be described together in a manner that is internally consistent, and consistent with current and future observations. The greatest advances have been steps towards this goal: the unification of terrestrial and celestial mechanics by Newton; of optics with the theories of electricity and magnetism by Maxwell; of geometry and the theory of gravitation by Einstein; of electromagnetism with weak interactions, but the work of unification can be completed if gravity is included. Experiments at the LHC and elsewhere should let us complete the Standard Model, but a unified theory will require first of all a solution of the conceptual problems and probably radically new ideas. Thus, motivation for our study is clear. Here we should like to understand the physical meaning of the fourth dimension. What does it means? The mathematical space is a smooth manifold. A smooth manifold or $C^{\infty}$-manifold is a differentiable manifold for which all the transition maps are smooth. That is, derivatives of all orders exist; so it is a $C^{k}$-manifold for all k. An atlas on the topological space $\mathrm{M}$ is a collection of pairs $\left(U_{\alpha}, \varphi_{\alpha}\right)$ called charts, where $U_{\alpha}$ are open sets that cover $\mathrm{M}$, and for each index $\alpha$

$$
\varphi_{\alpha}: U_{\alpha} \rightarrow R^{n}
$$

is a homeomorphism of $U_{\alpha}$ onto an open subset of n-dimensional real space. An equivalence class of such atlases is said to be a smooth structure. The underlying space $R^{n}$ is the space of n-tuples of real numbers $\left(q^{1}, q^{2}, \cdots, q^{n}\right)$ with usual topology. Here we should like to emphasize that coordinates $q^{1}, q^{2}, \cdots, q^{n}$ should be evidently considered on equal footing but space-time is a mathematical space whose points must be specified by both space and time coordinates. Hence, it is clear that there is no regular method to introduce space coordinates and time coordinate in the framework of a smooth manifold alone. Our goal is to recognize a regular transition from the mathematical space to space-time and with this derive new information about the nature of space and time. To this end, we consider the Dirac equation in the mathematical space. A comparison will be produced of the Dirac theory of the electron with spin in the simplest four- dimensional mathematical space and the original Dirac theory in the Minkowski space-time. New representations about nature of space, time, rotation, quark-lepton symmetry and confinement will be derived from this consideration.

\section{Dirac equation in a mathematical space}

Fundamental concepts, symmetries, and dynamical equations of the theory of dark matter are derived in [1] from the simple relation (the only first principle of a unified physics): everything in the concept of a mathematical space and a mathematical space in everything. It was shown that the electromagnetic field is the singlet state of the dark matter field and hence the last can be considered as a generalized electromagnetic field and a simple solution was given to the old problem of connecting the electomagnetic field with geometrical concept of parallel transport. To learn more about interactions between matter and dark matter on the microscopic level (and to recognize the fundamental role of internal symmetry in this case), the general covariant Dirac equation was derived and natural generelization was considered. In accordance with this consideration, the Dirac 
equation in the four-dimensional mathematical space reads

$$
i \gamma^{\mu} D_{\mu} \psi=m \psi
$$

where $D_{\mu}$ are linear differential operators

$$
D_{\mu}=E_{\mu}^{i} \partial_{i}=E_{\mu}^{1} \frac{\partial}{\partial q^{1}}+E_{\mu}^{2} \frac{\partial}{\partial q^{2}}+E_{\mu}^{3} \frac{\partial}{\partial q^{3}}+E_{\mu}^{4} \frac{\partial}{\partial q^{4}}
$$

and $E_{\mu}^{i}$ are a quadruplet of linear independent vector fields which puts in correspondence to the ground state of the generalized electromagnetic field and will be considered as of a frame field. The gamma matrices $\gamma^{\mu}$ are normalized as follows:

$$
\gamma^{\mu} \gamma^{v}+\gamma^{v} \gamma^{\mu}=2 \eta^{\mu v}, \quad \eta^{\mu v}=\eta_{\mu v}=\operatorname{diag}(1,-1,-1,-1)
$$

The world indices of vectors are denoted by latin letters $i, j, k, \cdots=1,2,3,4$, and the greek letters enumerate the gamma matrices and the vector fields in question $\mu, v \cdots=0,1,2,3$. For simplicity, we consider the case when the vector part of the srength tensor of the ground state

$$
U_{j k}^{i}=E_{\mu}^{i}\left(\partial_{j} E_{k}^{\mu}-\partial_{k} E_{j}^{\mu}\right)
$$

is equal to zero, $U_{i k}^{i}=0$. Here $E_{i}^{\mu}$ are components of four covector fields which one can construct purely algebraically from $E_{\mu}^{i}$, so that $E_{\mu}^{i} E_{j}^{\mu}=\delta_{l}^{i}$ holds valid.

Since

$$
\gamma^{\mu} D_{\mu}=\gamma^{0} D_{0}+\gamma^{1} D_{1}+\gamma^{2} D_{2}+\gamma^{3} D_{3},
$$

then to get a regular transition from the mathematical space to space-time, we need to introduce the system of coordinates $x^{1}, x^{2}, x^{3}, t$ in which the linear differential operator $D_{0}$ takes the form

$$
D_{0}=E_{0}^{i} \partial_{i} \rightarrow \frac{\partial}{\partial t}
$$

To this end, let us consider the system of ordinary differential equations

$$
\frac{d q^{i}}{d t}=E_{0}^{i}\left(q^{1}, q^{2}, q^{3}, q^{4}\right)
$$

It is well known that this system has a unique solution

$$
q^{1}=f_{1}\left(q_{0}^{1}, q_{0}^{2}, q_{0}^{3}, q_{0}^{4}, t\right), \cdots, q^{4}=f_{4}\left(q_{0}^{1}, q_{0}^{2}, q_{0}^{3}, q_{0}^{4}, t\right)
$$

which satisfies the condition

$$
q_{0}^{1}=f_{1}\left(q_{0}^{1}, q_{0}^{2}, q_{0}^{3}, q_{0}^{4}, t_{0}\right), \cdots, q_{0}^{4}=f_{4}\left(q_{0}^{1}, q_{0}^{2}, q_{0}^{3}, q_{0}^{4}, t_{0}\right) .
$$

Let the initial point $P\left(q_{0}^{1}, q_{0}^{2}, q_{0}^{3}, q_{0}^{4}\right)$ belongs to the $3 \mathrm{~d}$ surface $S$ which is parameterised by the coordinates $x^{1}, x^{2}, x^{3}$

$$
q_{0}^{1}=h_{1}\left(x^{1}, x^{2}, x^{3}\right), \quad q_{0}^{2}=h_{2}\left(x^{1}, x^{2}, x^{3}\right), \quad q_{0}^{3}=h_{3}\left(x^{1}, x^{2}, x^{3}\right), \quad q_{0}^{4}=h_{4}\left(x^{1}, x^{2}, x^{3}\right) .
$$


The surface $S$ should be chosen so that the variables $x^{1}, x^{2}, x^{3}, t$ define a new system of coordinates in the mathematical space and the tetrad take the following form:

$$
E_{0}^{i}=(0,0,0,1), \quad E_{1}^{i}=\left(E_{1}^{1}, E_{1}^{2}, E_{1}^{3}, 0\right), \quad E_{2}^{i}=\left(E_{2}^{1}, E_{2}^{2}, E_{2}^{3}, 0\right), \quad E_{3}^{i}=\left(E_{3}^{1}, E_{3}^{2}, E_{3}^{3}, 0\right) .
$$

The surface so defined will be called the characteristic surface of space-time, the variables $x^{1}, x^{2}, x^{3}$ will be called the space coordinates and accordingly the $t$ the time coordinate. We conclude that the space-time is a causal structure on the mathematical space which is defined by the vector field or congruence of lines. We recall that the congruence of lines is a set of lines characterized by that the only element of the set crosses every point of a manifold or its part. The lines belonging to the congruence do not intersect and fill either the whole manifold or its part. In the mathematical space equipped by the causal structure equation (1) takes the Hamiltonian form

$$
i \frac{\partial}{\partial t} \psi=H \psi
$$

where the operator $H$ does not contain the partial derivative up to $t$. After this general consideration we start to learn the simplest four- dimensional mathematical space $R^{4}$.

\section{Internal properties of the $4 \mathrm{~d}$ euclidian space}

Points of $R^{4}$ have the vector

$$
\mathbf{q}=\left(q^{1}, q^{2}, q^{3}, q^{4}\right)
$$

and the quaternion representations

$$
q=q^{1} i+q^{2} j+q^{3} k+q^{4} 1,
$$

with the usual linear structure. The quaternion algebra is defined as usual

$$
i^{2}=j^{2}=k^{2}=-1, \quad i j=-j i=k, j k=-k j=i, k i=-i k=j .
$$

The scalar product

$$
\mathbf{p} \cdot \mathbf{q}=p^{1} q^{1}+p^{2} q^{2}+p^{3} q^{3}+p^{4} q^{4}
$$

can be written in the quaternion form in two ways

$$
\mathbf{p} \cdot \mathbf{q}=\frac{1}{2}(p \bar{q}+q \bar{p})=\frac{1}{2}(\bar{p} q+\bar{q} p)
$$

where $\bar{q}=-q^{1} i-q^{2} j-q^{3} k+q^{4} 1$. The scalar product is invariant with respect to the right and left turn dilatations

$$
q \Rightarrow \tilde{q}=s q, \quad \Rightarrow \tilde{q}=q \bar{t}
$$

since

$$
\tilde{\mathbf{p}} \cdot \tilde{\mathbf{q}}=s \bar{s}(\mathbf{p} \cdot \mathbf{q}), \quad \tilde{\mathbf{p}} \cdot \tilde{\mathbf{q}}=t \bar{t}(\mathbf{p} \cdot \mathbf{q}) .
$$

We suppose that $q$ and $\lambda q$, where $\lambda$ is number, are equivalent. For a given $\mathrm{q}$, equations $q=$ $s q, \quad q=q \bar{t}$ have only trivial solutions $s=\bar{t}=1$ and the absence of fixed points under turn dilatations exhibits a fundamental property of the space in question: the existence of two simply transitive groups of transformations. 
Now we introduce two natural frames intrinsically connected with the space in question. The standard frame

$$
\begin{gathered}
\mathbf{c}_{1}=(1,0,0,0) \quad \mathbf{c}_{2}=(0,1,0,0) \quad \mathbf{c}_{3}=(0,0,1,0) \quad \mathbf{c}_{4}=(0,0,0,1), \\
c_{1}=i, c_{2}=j, c_{3}=k, c_{4}=1
\end{gathered}
$$

gives rise to the pair of right-handled moving frames

$$
\begin{aligned}
& m_{1}=i q, m_{2}=j q, m_{3}=k q, m_{4}=1 q, \quad n_{1}=q i, n_{2}=q j, n_{3}=q k, n_{4}=q 1 . \\
& \mathbf{m}_{1}=\left(q^{4},-q^{3}, q^{2},-q^{1}\right) \\
& \mathbf{m}_{2}=\left(q^{3}, \quad q^{4},-q^{1},-q^{2}\right) \\
& \mathbf{m}_{3}=\left(\begin{array}{llll}
-q^{2}, & q^{1}, & q^{4}, & -q^{3}
\end{array}\right) \\
& \mathbf{m}_{4}=\left(\begin{array}{cccc}
q^{1}, & q^{2}, & q^{3}, & q^{4}
\end{array}\right) \\
& \mathbf{n}_{1}=\left(q^{4}, q^{3},-q^{2},-q^{1}\right) \\
& \mathbf{n}_{2}=\left(\begin{array}{lll}
-q^{3}, & q^{4}, & q^{1},-q^{2}
\end{array}\right) \\
& \mathbf{n}_{3}=\left(q^{2},-q^{1}, q^{4},-q^{3}\right) \\
& \mathbf{n}_{4}=\left(\begin{array}{cccc}
q^{1}, & q^{2}, & q^{3}, & q^{4}
\end{array}\right)
\end{aligned}
$$

It is easy to see that

$$
\mathbf{m}_{a} \cdot \mathbf{m}_{b}=q \bar{q} \delta_{a b}, \quad \mathbf{n}_{a} \cdot \mathbf{n}_{b}=q \bar{q} \delta_{a b}, \quad(a, b=1,2,3,4) .
$$

Let us consider the running point $T\left(q_{1}, q_{2}, q_{3}, q_{4}\right)$, and the twelve coherent points

$$
\begin{array}{ccc}
A\left(q^{4},-q^{3}, q^{2},-q^{1}\right), & B\left(q^{3}, q^{4},-q^{1},-q^{2}\right), & C\left(-q^{2}, q^{1}, q^{4},-q^{3}\right), \\
K\left(q^{4}, q^{3},-q^{2},-q^{1}\right), & L\left(-q^{3}, q^{4}, q^{1},-q^{2}\right), & M\left(q^{2},-q^{1}, q^{4},-q^{3}\right), \\
\bar{A}\left(-q^{4}, q^{3},-q^{2}, q^{1}\right), & \bar{B}\left(-q^{3},-q^{4}, q^{1}, q^{2}\right), & \bar{C}\left(q^{2},-q^{1},-q^{4}, q^{3}\right), \\
\bar{K}\left(-q^{4},-q^{3}, q^{2}, q^{1}\right), & \bar{L}\left(q^{3},-q^{4},-q^{1}, q^{2}\right), & \bar{M}\left(-q^{2}, q^{1},-q^{4}, q^{3}\right) .
\end{array}
$$

The distance function is defined as usual

$$
d_{P Q}^{2}=\left(p^{1}-q^{1}\right)^{2}+\left(p^{2}-q^{2}\right)^{2}+\left(p^{3}-q^{3}\right)^{2}+\left(p^{4}-q^{4}\right)^{2} .
$$

With this it is easy to see that

$$
\begin{aligned}
& d_{A B}^{2}=d_{A C}^{2}=d_{B C}^{2}=d_{T A}^{2}=d_{T B}^{2}=d_{T C}^{2}=2 q \bar{q}, \\
& d_{\bar{A} \bar{B}}^{2}=d_{\bar{A} \bar{C}}^{2}=d_{\bar{B} \bar{C}}^{2}=d_{T \bar{A}}^{2}=d_{T \bar{B}}^{2}=d_{T \bar{C}}^{2}=2 q \bar{q}
\end{aligned}
$$

and

$$
\begin{aligned}
& d_{K L}^{2}=d_{K M}^{2}=d_{L M}^{2}=d_{T K}^{2}=d_{T L}^{2}=d_{T M}^{2}=2 q \bar{q}, \\
& d_{\bar{K} \bar{L}}^{2}=d_{\bar{K} \bar{M}}^{2}=d_{\bar{L} \bar{M}}^{2}=d_{T \bar{K}}^{2}=d_{T \bar{L}}^{2}=d_{T \bar{M}}^{2}=2 q \bar{q},
\end{aligned}
$$


where $d_{A B}$ is the distance between the points $A$ and $B$. We see a pair of regular tetrahedrons and a dual one with a common vertex $T: T A B C$ and $T K L M, T \bar{A} \bar{B} \bar{C}$ and $T \bar{K} \bar{L} \bar{M}$. These tetrahedrons give a visual representation of the frames in question

$$
\mathbf{m}_{\mu} \quad \mathbf{n}_{\mu}, \quad-\mathbf{m}_{\mu} \quad-\mathbf{n}_{\mu}, \quad(\mu=1,2,3)
$$

and discover the nature of rotational motion. Let $\mathbf{q}=\mathbf{q}(t)$ be a trajectory in $R^{4}$. When point $T$ moves along this trajectory, the tetrahedrons $T A B C$ and $T K L M$ are pulsed and rotated with respect to each other. And the same for the dual tetrahedrons $T \bar{A} \bar{B} \bar{C}$ and $T \bar{K} \bar{L} \bar{M}$.

The matrix of scalar products

$$
\mathrm{P}_{\mu \nu}=\mathbf{m}_{\mu} \cdot \mathbf{n}_{v}, \quad(\mu, v=1,2,3)
$$

describes this relative rotational motion.

The scalar products of the tangent vector $\dot{\mathbf{q}}=d \mathbf{q} / d t$ with the vectors of dual frames $\mathbf{m}_{a}$ and $\mathbf{n}_{a},(a=1,2,3,4)$

$$
\begin{gathered}
\mathbf{m}_{1} \cdot \frac{d \mathbf{q}}{d t}=q^{4} \frac{d q^{1}}{d t}-q^{3} \frac{d q^{2}}{d t}+q^{2} \frac{d q^{3}}{d t}-q^{1} \frac{d q^{4}}{d t} \\
\mathbf{m}_{2} \cdot \frac{d \mathbf{q}}{d t}=q^{3} \frac{d q^{1}}{d t}+q^{4} \frac{d q^{2}}{d t}-q^{1} \frac{d q^{3}}{d t}-q^{2} \frac{d q^{4}}{d t}, \quad \mathbf{m}_{3} \cdot \frac{d \mathbf{q}}{d t}=-q^{2} \frac{d q^{1}}{d t}+q^{1} \frac{d q^{2}}{d t}+q^{4} \frac{d q^{3}}{d t}-q^{3} \frac{d q^{4}}{d t} \\
\mathbf{n}_{1} \cdot \frac{d \mathbf{q}}{d t}=q^{4} \frac{d q^{1}}{d t}+q^{3} \frac{d q^{2}}{d t}-q^{2} \frac{d q^{3}}{d t}-q^{1} \frac{d q^{4}}{d t} \\
\mathbf{n}_{2} \cdot \frac{d \mathbf{q}}{d t}=-q^{3} \frac{d q^{1}}{d t}+q^{4} \frac{d q^{2}}{d t}+q^{1} \frac{d q^{3}}{d t}-q^{2} \frac{d q^{4}}{d t}, \quad \mathbf{n}_{3} \cdot \frac{d \mathbf{q}}{d t}=q^{2} \frac{d q^{1}}{d t}-q^{1} \frac{d q^{2}}{d t}+q^{4} \frac{d q^{3}}{d t}-q^{3} \frac{d q^{4}}{d t} \\
\mathbf{m}_{4} \cdot \frac{d \mathbf{q}}{d t}=\mathbf{n}_{4} \cdot \frac{d \mathbf{q}}{d t}=q^{1} \frac{d q^{1}}{d t}+q^{2} \frac{d q^{2}}{d t}+q^{3} \frac{d q^{3}}{d t}+q^{4} \frac{d q^{4}}{d t}
\end{gathered}
$$

are invariant with respect to the left and right turn dilatations. The invariants

$$
\Omega_{\mu}=\frac{1}{2} \mathbf{m}_{\mu} \cdot \frac{d \mathbf{q}}{d t}, \quad \tilde{\Omega}_{\mu}=\frac{1}{2} \mathbf{n}_{\mu} \cdot \frac{d \mathbf{q}}{d t}, \quad(\mu=1,2,3)
$$

are components of angular velocity of rotation of tetrahedron $T A B C$ with respect to tetrahedron $T K L M$ and vice versa. These invariants play the important role in the rigid- body dynamics as well. Thus, a kinematics of rotational motion has an adequate representation in the four dimensions.

To quantize the rotational motion, let us introduce the $4 d$ operator $\nabla$

$$
\nabla_{4}=\left(\frac{\partial}{\partial q^{1}}, \frac{\partial}{\partial q^{2}}, \frac{\partial}{\partial q^{3}}, \frac{\partial}{\partial q^{4}},\right)
$$

and setting

$$
M_{v}=\frac{1}{2}\left(\mathbf{m}_{v} \cdot \nabla_{4}\right), \quad N_{v}=\frac{1}{2}\left(\mathbf{n}_{v} \cdot \nabla_{4}\right), \quad(v=1,2,3)
$$

we have six antihermitian operators of angular momentum of a rotational motion. Factor $\frac{1}{2}$ is essential since natural commutation relations hold valid 


$$
M_{1} M_{2}-M_{2} M_{1}=M_{3}, \quad N_{1} N_{2}-N_{2} N_{1}=-N_{3}
$$

and so on. The operator of dilatations

$$
D=\left(\mathbf{m}_{4} \cdot \nabla_{4}\right)=q^{1} \frac{\partial}{\partial q^{1}}+q^{2} \frac{\partial}{\partial q^{2}}+q^{3} \frac{\partial}{\partial q^{3}}+q^{4} \frac{\partial}{\partial q^{4}}
$$

has important meaning as well since it commutes with the operators of angular momentum

$$
D M_{v}-M_{v} D=0, \quad D N_{v}-N_{v} D=0, \quad(v=1,2,3)
$$

and will play a key role in the description of the rotational motion on the quantum level. Now we shall introduce two natural frame fields in the space in question and consider the Dirac equations associated with these quadruplets of linear independent vector fields.

\section{Global frame field}

Let

$$
\mathbf{a}=\left(a^{1}, a^{2}, a^{3}, a^{4}\right)
$$

be a constant unit vector, then a global natural frame field in $R^{4}$ is defined as follows

$$
\begin{gathered}
\mathbf{E}_{0}=\left(a^{1}, a^{2}, a^{3}, a^{4}\right), \quad \mathbf{E}_{1}=\left(-a^{4},-a^{3}, a^{2}, a^{1}\right), \\
\mathbf{E}_{2}=\left(a^{3},-a^{4},-a^{1}, a^{2}\right), \quad \mathbf{E}_{3}=\left(-a^{2}, a^{1},-a^{4}, a^{3}\right) .
\end{gathered}
$$

We put

$$
D_{0}=\mathbf{E}_{0} \cdot \nabla_{4}, \quad D_{1}=\mathbf{E}_{1} \cdot \nabla_{4}, \quad D_{2}=\mathbf{E}_{2} \cdot \nabla_{4}, \quad D_{3}=\mathbf{E}_{3} \cdot \nabla_{4}
$$

then the Dirac equation in the 4d Euclidian space reads

$$
i \gamma^{\mu} D_{\mu} \psi=\frac{m c}{\hbar} \psi
$$

Since

$$
\gamma^{\mu} D_{\mu}=\gamma^{0} D_{0}+\gamma^{1} D_{1}+\gamma^{2} D_{2}+\gamma^{3} D_{3}
$$

then to get a regular transition from the Dirac equation in question to the original Dirac equation, we need to introduce the system of coordinates $x^{1}, x^{2}, x^{3}, t$ in which the linear differential operator $D_{0}$ takes the following form:

$$
D_{0}=E_{0}^{i} \partial_{i}=a^{i} \partial_{i} \rightarrow \frac{\partial}{\partial t}
$$

To this end (see section two) we need to solve the system of equations

$$
\frac{d q^{i}}{d t}=a^{i}
$$

The general solution is a straight line that goes through the fixed point $\mathbf{q}_{0}=\left(q_{0}^{1}, q_{0}^{2}, q_{0}^{3}, q_{0}^{4}\right)$ :

$$
\mathbf{q}(t)=\mathbf{a}\left(t-t_{0}\right)+\mathbf{q}_{0} .
$$


We define the $3 \mathrm{~d}$ characteristic surface $S$ in the space of initial data as follows:

$$
\mathbf{a} \cdot \mathbf{q}_{0}=t_{0} .
$$

The general solution to equation (6) has the form

$$
\mathbf{q}_{0}=t_{0} \mathbf{E}_{0}+x \mathbf{E}_{1}+y \mathbf{E}_{2}+z \mathbf{E}_{3} .
$$

Substituting this representation into formula (5) we have

$$
\mathbf{q}=t \mathbf{E}_{0}+x \mathbf{E}_{1}+y \mathbf{E}_{2}+z \mathbf{E}_{3} .
$$

The Dirac equation in the coordinates $t, x, y, z$ has the ordinary form

$$
i\left(\gamma^{0} \frac{\partial}{\partial t}+\gamma^{1} \frac{\partial}{\partial x}+\gamma^{2} \frac{\partial}{\partial y}+\gamma^{3} \frac{\partial}{\partial z}\right) \psi=\frac{m c}{\hbar} \psi
$$

One can work in either the coordinates $q^{1}, q^{2}, q^{3}, q^{4}$ (that are considered on equal footing) or the coordinates $t, x, y, z$ but the first approach looks like more fundamental because the direction of the vector $\mathbf{a}$ is not fixed, and this distinctive degeneration is not visible in the second approach.

Now it is important to show the definition of interval in the $4 \mathrm{~d}$ Euclidian space. The interval in $R^{4}$ is defined as follows. Let

$$
\mathbf{q}_{s}=2 \mathbf{a}(\mathbf{a} \cdot \mathbf{q})-\mathbf{q}
$$

be the vector symmetrical to the vector $\mathbf{q}$ with respect to the vector $\mathbf{a}$. Then in the coordinates $q^{1}, q^{2}, q^{3}, q^{4}$ the interval can be written as follows:

$$
s^{2}=\mathbf{q} \cdot \mathbf{q}_{s}=2(\mathbf{a} \cdot \mathbf{q})^{2}-\mathbf{q} \cdot \mathbf{q}=(\mathbf{q} \cdot \mathbf{q}) \cos 2 \theta,
$$

where $\theta$ is an angle between $\mathbf{a}$ and $\mathbf{q}$. It is easy to see that in the coordinates $t, x, y, z$,

$$
s^{2}=t^{2}-x^{2}-y^{2}-z^{2}
$$

We see that the existence of a natural global frame in the $4 \mathrm{~d}$ Euclidian space and the bilateral symmetry presupposes the existence of Minkowski space-time and hence the known causal structure discovered here as a preferred system of coordinates defined by the given direction in the $4 \mathrm{~d}$ Euclidian space. The causal structure may be considered in this case as spontaneous breaking of isotropy of the four- dimensional Euclidian space. The global frame field defines a metric as usual

$$
g_{i j}=\eta_{\mu v} E_{i}^{\mu} E_{j}^{v}=2 a_{i} a_{j}-\delta_{i j} .
$$

\section{Local frame field}

Let

$$
\mathbf{q}=\left(q^{1}, q^{2}, q^{3}, q^{4}\right)
$$

be a radius-vector, then a natural local frame field in the $4 \mathrm{~d}$ Euclidian space can be represented as a quadruplet of orthogonal unit vector fields

$$
\mathbf{E}_{0}=\left(\frac{q^{1}}{\tau}, \frac{q^{2}}{\tau}, \frac{q^{3}}{\tau}, \frac{q^{4}}{\tau}\right), \quad \mathbf{E}_{1}=\left(\frac{-q^{4}}{\tau}, \frac{-q^{3}}{\tau}, \frac{q^{2}}{\tau}, \frac{q^{1}}{\tau}\right),
$$




$$
\mathbf{E}_{2}=\left(\frac{q^{3}}{\tau}, \frac{-q^{4}}{\tau}, \frac{-q^{1}}{\tau}, \frac{q^{2}}{\tau}\right), \quad \mathbf{E}_{3}=\left(\frac{-q^{2}}{\tau}, \frac{q^{1}}{\tau}, \frac{-q^{4}}{\tau}, \frac{q^{3}}{\tau}\right),
$$

where

$$
\tau=\sqrt{\mathbf{q} \cdot \mathbf{q})}=\sqrt{\left(q^{1}\right)^{2}+\left(q^{2}\right)^{2}+\left(q^{3}\right)^{2}+\left(q^{4}\right)^{2}}
$$

is the length of the radius-vector. The metric defined by the local frame field has a simple representation $g_{i j}=\eta_{\mu v} E_{i}^{\mu} E_{j}^{v}=2 t_{i} t_{j}-\delta i j$. We again put

$$
D_{0}=\mathbf{E}_{0} \cdot \nabla, \quad D_{1}=\mathbf{E}_{1} \cdot \nabla, \quad D_{2}=\mathbf{E}_{2} \cdot \nabla, \quad D_{3}=\mathbf{E}_{3} \cdot \nabla
$$

but here the operator $\nabla$ is defined as follows:

$$
\nabla=\nabla_{4}-\frac{3}{2 \tau^{2}} \mathbf{q}
$$

since the vector part of the tensor $U_{j k}^{i}$, defined above, is not equal to zero in this case. The Dirac equation describing the rotational motion on the quantum level takes the following form:

$$
i \gamma^{\mu} D_{\mu} \psi=\frac{m c}{\hbar} \psi
$$

Let us consider how to equip the $4 \mathrm{~d}$ Euclidian space with a preferred system of coordinates (a space-time structure) in the case of the rotational motion. The general solution of the system of equations

$$
\frac{d q^{i}}{d \tau}=\frac{q^{i}}{\sqrt{\left(q^{1}\right)^{2}+\left(q^{2}\right)^{2}+\left(q^{3}\right)^{2}+\left(q^{4}\right)^{2}}}
$$

can be written in two ways

$$
q^{i}(\tau)=q_{0}^{i} \frac{\tau}{\tau_{0}}, \quad \tau \in\left[\tau_{0}, \infty\right)
$$

where the initial data belong to the $3 \mathrm{~d}$ sphere

$$
\mathbf{q}_{0} \cdot \mathbf{q}_{0}=\tau_{0}^{2} .
$$

and

$$
q^{i}(\tau)=q_{0}^{i} \tau, \quad \tau \in[0, \infty)
$$

where the initial data belong to the unit $3 \mathrm{~d}$ sphere

$$
\mathbf{q}_{0} \cdot \mathbf{q}_{0}=1 .
$$

The characteristic surface in this case can be parameterized by the Euler angles, $\theta, \varphi, \gamma$ In the coordinates $\tau, \theta, \varphi, \gamma$, we have

$$
\begin{gathered}
D_{0}=\frac{\partial}{\partial \tau}-\frac{3}{2 \tau}, \quad D_{1}=\frac{1}{\tau}\left(-\cot \theta \cos \gamma \frac{\partial}{\partial \gamma}-\sin \gamma \frac{\partial}{\partial \theta}+\frac{\cos \gamma}{\sin \theta} \frac{\partial}{\partial \varphi}\right), \\
D_{2}=\frac{1}{\tau}\left(-\cot \theta \sin \gamma \frac{\partial}{\partial \gamma}+\cos \gamma \frac{\partial}{\partial \theta}+\frac{\sin \gamma}{\sin \theta} \frac{\partial}{\partial \varphi}\right), \quad D_{3}=\frac{1}{\tau} \frac{\partial}{\partial \gamma} .
\end{gathered}
$$

Let us pay attention to the following internal properties of the four-dimensional Euclidian space: kinematical picture and nature of rotational motion; existence of two space-time or causal 
structures. In one case, the space-time structure may be geometrically represented as the congruence of parallel $3 \mathrm{~d}$ planes and the congruence of parallel straight lines orthogonal to these planes. In the other case, the space-time structure is defined as the congruence of the $3 \mathrm{~d}$ sphere with a common centre and the congruence of rays orthogonal to the $3 \mathrm{~d}$ spheres. A physical interpretation: we put forward the idea that the behavior of leptons is defined by the first causal structure, and the physics of quarks is tightly connected with the new causal structure, which represents a rotating matter. The latter provides understanding and rational proof of quark-lepton symmetry, quark confinement (confinement is not a force because in any case there is a more powerful one),conservation of the so called baryon number (read new causal structure). Equations (4), (7) and machinery of the electroweak theory provide the new theoretical basis for understanding of the world of leptons and quarks.

The action for the point particle associated with the rotational motion can be written in the following form:

$$
S=-m c \int_{p}^{q} \sqrt{1-\tau^{2} \omega^{2}} d \tau,
$$

where $\omega=d l / d \tau$ and $d l$ is the element of the arc on the unit $3 d$ sphere. Really, $\overrightarrow{d u} \cdot \overrightarrow{d u}=$ $d \tau^{2}+\tau^{2} d l^{2}$, and $\vec{u} \cdot \overrightarrow{d u}=\tau d \tau$. On this ground one can develop the classical mechanics in the new frameworks.

In conclusion of this section, we formulate the Maxwell equations in the framework of the new causal structure. Let $A_{i}$ be the vector potential of the electromagnetic field. The gauge invariant tensor of the electromagnetic field is defined as usual $F_{i j}=\partial_{i} A_{j}-\partial_{j} A_{i}$. The strength of the electric field is a general covariant and gauge invariant quantity that is defined by the equation $E_{i}=t^{k} F_{i k}$, where in our case $t^{k}=t_{k}=q^{k} / \tau$.

The rotor of the vector field $\mathbf{A}=\left(A_{1}, A_{2}, A_{3}, A_{4}\right)$ is defined as a vector product of $\nabla_{4}$ and $\mathbf{A}$

$$
\operatorname{rot} \mathbf{A}=\nabla_{4} \times \mathbf{A}, \quad(\operatorname{rot} \mathbf{A})^{i}=e^{i j k l} t_{j} \partial_{k} A_{l}=\frac{1}{2} e^{i j k l} t_{j}\left(\partial_{k} A_{l}-\partial_{l} A_{k}\right),
$$

where $e^{i j k l}$ are the contravariant components of the Levi-Civita tensor normalized as $e_{1234}=1$. The general covariant and gauge invariant definition of the magnetic field strength is given by the formula $\mathbf{H}=\operatorname{rot} \mathbf{A}, \quad H^{i}=(\operatorname{rot} \mathbf{A})^{i}$. Thus, $H_{i}=t^{k} \stackrel{*}{F}_{i k}$, where $\stackrel{*}{F}_{i j}=g_{i k} g_{j l} F^{k l}=\frac{1}{2} g_{i k} g_{j l} e^{k l m n} F_{m n}$. It is evident that vectors $\mathbf{E}$ and $\mathbf{H}$ are orthogonal to $\mathbf{q}$

$$
\mathbf{q} \cdot \mathbf{E}=0, \quad \mathbf{q} \cdot \mathbf{H}=0 .
$$

Below, the Maxwell equations are written in the form that is most suitable for solution:

$$
\begin{gathered}
\left(\mathbf{D}_{0} \cdot \nabla_{4}\right) \mathbf{H}+\frac{2}{\tau} \mathbf{H}=-\operatorname{rot} \mathbf{E}, \\
\left(\mathbf{D}_{0} \cdot \nabla_{4}\right) \mathbf{E}+\frac{2}{\tau} \mathbf{E}=\operatorname{rot} \mathbf{H}+e \mathbf{J}, \\
\nabla_{4} \cdot \mathbf{E}=e \bar{\psi} \gamma^{0} \psi, \quad \nabla_{4} \cdot \mathbf{H}=0,
\end{gathered}
$$

where the current $\mathbf{J}$ is given by the expression

$$
\mathbf{J}=\mathbf{E}_{1} \bar{\psi} \gamma^{1} \psi+\mathbf{E}_{2} \bar{\psi} \gamma^{2} \psi+\mathbf{E}_{3} \bar{\psi} \gamma^{3} \psi
$$




\section{Connection with three dimensional space}

To complete the picture of rotational motion and throw light on some other questions, we consider here properties of natural mappings of the four-dimensional Euclidian space onto the three-dimensional one. Let $\varphi(x, y, z)$ be a differentiable function of the cartesian coordinates $x, y, z$ of the three-dimensional euclidian space and three differentiable functions

$$
x=x\left(q^{1}, q^{2}, q^{3}, q^{4}\right), \quad y=y\left(q^{1}, q^{2}, q^{3}, q^{4}\right), \quad z=z\left(q^{1}, q^{2}, q^{3}, q^{4}\right)
$$

define a mapping of $R^{4}$ onto $E^{3}$. Let us calculate the result of the action of the linear differential operator

$$
L=\xi^{i}\left(q^{1}, q^{2}, q^{3}, q^{4}\right) \frac{\partial}{\partial q^{i}}
$$

on the function $\varphi(x, y, z)$. Using the chain rule we have

$$
L \varphi(x, y, z)=(L x) \frac{\partial \varphi}{\partial x}+(L y) \frac{\partial \varphi}{\partial y}+(L z) \frac{\partial \varphi}{\partial z} .
$$

If functions

$$
L x=\xi^{i} \frac{\partial x}{\partial q^{i}}, \quad L y=\xi^{i} \frac{\partial y}{\partial q^{i}}, \quad L z=\xi^{i} \frac{\partial z}{\partial q^{i}}
$$

of the variables $q^{1}, q^{2}, q^{3}, q^{4}$ can be presented as functions of the variables $x, y, z$, then setting

$$
L x=v_{x}(x, y, z), \quad L y=v_{y}(x, y, z), \quad L z=v_{z}(x, y, z),
$$

one can calculate the result of the action of the operator $L$ with the help of the new differential operator

$$
V=v_{x} \frac{\partial}{\partial x}+v_{y} \frac{\partial}{\partial y}+v_{z} \frac{\partial}{\partial z}
$$

which can be considered as transform of the operator $L$ under the mapping in question. After these general remarks let us consider natural mapping of $R^{4}$ onto $E^{3}$. It is well known that a rotation with dilatation of the vector $v=v_{1} i+v_{2} j+v_{3} k$ can be presented as follows:

$$
v \rightarrow s v \bar{s}
$$

Let us consider the quaternions

$$
\begin{array}{lll}
R_{1}=q i \bar{q}, & R_{2}=q j \bar{q}, & R_{3}=q k \bar{q}, \\
T_{1}=\bar{q} i q, & T_{2}=\bar{q} j q, & T_{3}=\bar{q} k q .
\end{array}
$$

Under the left turn dilatations $q \rightarrow s q$, the quaternions $R_{1}, R_{2}, R_{3}$, transform as follows $R_{\mu} \rightarrow$ $s R_{\mu} \bar{s}, \quad(\mu=1,2,3)$. Under the right turn dilatations $q \rightarrow q \bar{t}$, the quaternions $T_{1}, T_{2}, T_{3}$ transform similarly to $R_{\mu}, T_{\mu} \rightarrow t T_{\mu} \bar{t}, \quad(\mu=1,2,3)$. We see that the coordinates of the quaternions in question can be considered as cartesian coordinates of $E^{3}$. We denote these coordinates as $x_{\mu}, y_{\mu}, z_{\mu},(\mu=1,2,3)$ and, respectively, $\xi_{\mu}, \eta_{\mu}, \zeta_{\mu},(\mu=1,2,3)$,

$$
\mathbf{R}_{\mu}=\left(x_{\mu}, y_{\mu}, z_{\mu}\right), \quad \mathbf{T}_{\mu}=\left(\xi_{\mu}, \eta_{\mu}, \zeta_{\mu}\right) .
$$


The vectors $\mathbf{R}_{\mu}$, and $\mathbf{T}_{\mu}$ have the same length and constitute the right-handled orthogonal bases since

$$
\mathbf{R}_{1} \times \mathbf{R}_{2}=q \bar{q} \mathbf{R}_{3}, \quad \mathbf{R}_{1} \cdot\left(\mathbf{R}_{2} \times \mathbf{R}_{3}\right)=(q \bar{q})^{3}, \quad \mathbf{T}_{1} \times \mathbf{T}_{2}=q \bar{q} \mathbf{T}_{3}, \quad \mathbf{T}_{1} \cdot\left(\mathbf{T}_{2} \times \mathbf{T}_{3}\right)=(q \bar{q})^{3} .
$$

Here we are slightly detained to give a simple and important geometrical interpretation of Cartan spinors [2],[3], which is tightly connected with the complex-analytic structures on $R^{4}$. To this end, let us consider the complex null vectors

$$
\mathbf{W}_{1}=\mathbf{R}_{2}+\sqrt{-1} \mathbf{R}_{3}, \quad \mathbf{W}_{2}=\mathbf{R}_{3}+\sqrt{-1} \mathbf{R}_{1}, \quad \mathbf{W}_{3}=\mathbf{R}_{1}+\sqrt{-1} \mathbf{R}_{2} .
$$

Calculating components of these vectors we have

$$
\mathbf{W}_{1}=\left(u_{1}, v_{1}, w_{1},\right)=\left(2 \xi_{1} \xi_{2}, \quad \xi_{1}^{2}-\xi_{2}^{2}, \quad-\sqrt{-1} \xi_{1}^{2}-\sqrt{-1} \xi_{2}^{2}\right),
$$

where $\xi_{1}=q^{2}+\sqrt{-1} q^{3}, \quad \xi_{2}=q^{1}+\sqrt{-1} q^{4}$,

$$
\mathbf{W}_{2}=\left(u_{2}, v_{2}, w_{2},\right)=\left(-\sqrt{-1} \eta_{1}^{2}-\sqrt{-1} \eta_{2}^{2}, \quad 2 \eta_{1} \eta_{2}, \quad \eta_{1}^{2}-\eta_{2}^{2}\right)
$$

where $\eta_{1}=q^{3}+\sqrt{-1} q^{1}, \quad \eta_{2}=q^{2}+\sqrt{-1} q^{4}$,

$$
\mathbf{W}_{3}=\left(u_{3}, v_{3}, w_{3},\right)=\left(\zeta_{1}^{2}--\zeta_{2}^{2}, \quad-\sqrt{-1} \zeta_{1}^{2}-\sqrt{-1} \zeta_{2}^{2}, \quad 2 \zeta_{1} \zeta_{2}\right)
$$

where $\zeta_{1}=q^{1}+\sqrt{-1} q^{2}, \quad \zeta_{2}=q^{3}+\sqrt{-1} q^{4}$.

Studying the behavior of the pairs $\left(\xi_{1}, \xi_{2}\right), \quad\left(\eta_{1}, \eta_{2}\right), \quad\left(\zeta_{1}, \zeta_{2}\right)$ under the turn dilatations we conclude that these pairs are spinors. It is also evident that Cartan spinor is simply the system of complex coordinates on $R^{4}$. The general theory of complex manifolds is explained in [4]. Actually, it is shown that there are three canonical systems of complex coordinates defined by the complex structures $i, j, k$. The turn dilatations in the complex coordinates coinside with spinor transformations. To introduce the spinor with the so called dotted indices, one simply needs to consider the vectors

$$
\overline{\mathbf{W}}_{1}=\mathbf{R}_{2}-\sqrt{-1} \mathbf{R}_{3}, \quad \overline{\mathbf{W}}_{2}=\mathbf{R}_{3}-\sqrt{-1} \mathbf{R}_{1}, \quad \overline{\mathbf{W}}_{3}=\mathbf{R}_{1}-\sqrt{-1} \mathbf{R}_{2} .
$$

Thus, it is evident that the spinors do not represent a new geometrical quantity.

Now it is time to prolong and write out expressions for the coordinates $x_{\mu}, y_{\mu}, z_{\mu},(\mu=1,2,3)$ and $\xi_{\mu}, \eta_{\mu}, \zeta_{\mu},(\mu=1,2,3)$. We have

$$
\begin{gathered}
x_{1}=\left(q^{1}\right)^{2}-\left(q^{2}\right)^{2}-\left(q^{3}\right)^{2}+\left(q^{4}\right)^{2}, \quad y_{1}=2 q^{1} q^{2}+2 q^{3} q^{4}, \quad z_{1}=2 q^{1} q^{3}-2 q^{2} q^{4}, \\
x_{2}=2 q^{1} q^{2}-2 q^{3} q^{4}, \quad y_{2}=-\left(q^{1}\right)^{2}+\left(q^{2}\right)^{2}-\left(q^{3}\right)^{2}+\left(q^{4}\right)^{2}, \quad z_{2}=2 q^{1} q^{4}+2 q^{2} q^{3}, \\
x_{3}=2 q^{1} q^{3}+2 q^{2} q^{4}, \quad y_{3}=-2 q^{1} q^{4}+2 q^{2} q^{3}, \quad z_{3}=-\left(q^{1}\right)^{2}-\left(q^{2}\right)^{2}+\left(q^{3}\right)^{2}+\left(q^{4}\right)^{2} .
\end{gathered}
$$

and

$$
\left(\xi_{1}, \eta_{1}, \zeta_{1}\right)=\left(x_{1}, x_{2}, x_{3}\right), \quad\left(\xi_{2}, \eta_{2}, \zeta_{2}\right)=\left(y_{1}, y_{2}, y_{3}\right), \quad\left(\xi_{3}, \eta_{3}, \zeta_{3}\right)=\left(z_{1}, z_{2}, z_{3}\right) .
$$

Thus, all natural mappings of $R^{4}$ onto $E^{3}$ are presented. Now it is interesting to find transforms of the operators of the angular momenta of the rotational motion. Below, the results of calculations 
will be presented only for one case (with comments only with respect to other situations). For obviousness, let us put $x_{1}=x, \quad y_{1}=y, \quad z_{1}=z$. After some calculations the following results can be presented:

$$
\begin{gathered}
M_{1} \varphi(x, y, z)=0 \\
-M_{2} \varphi(x, y, z)=x_{3} \frac{\partial \varphi}{\partial x}+y_{3} \frac{\partial \varphi}{\partial y}+z_{3} \frac{\partial \varphi}{\partial z}, \quad M_{3} \varphi(x, y, z)=x_{2} \frac{\partial \varphi}{\partial x}+y_{2} \frac{\partial \varphi}{\partial y}+z_{2} \frac{\partial \varphi}{\partial z} .
\end{gathered}
$$

It is visible that the operators in question have no transforms. In the other case a picture is more interesting since

$$
\begin{gathered}
N_{1} \varphi(x, y, z)=0 \frac{\partial \varphi}{\partial x}-z \frac{\partial \varphi}{\partial y}+y \frac{\partial \varphi}{\partial z} \\
N_{2} \varphi(x, y, z)=z \frac{\partial \varphi}{\partial x}+0 \frac{\partial \varphi}{\partial y}-x \frac{\partial \varphi}{\partial z}, \quad N_{3} \varphi(x, y, z)=-y \frac{\partial \varphi}{\partial x}+x \frac{\partial \varphi}{\partial y}+0 \frac{\partial \varphi}{\partial z} .
\end{gathered}
$$

Let us put $\mathbf{N}=\left(N_{1}, N_{2}, N_{3}\right.$. $)$ and the last relations can be written as follows:

$$
\mathbf{N} \varphi(x, y, z)=(\mathbf{r} \times \nabla) \varphi(x, y, z) .
$$

These relations are valid for all coordinates $x_{\mu}, y_{\mu}, z_{\mu},(\mu=1,2,3)$. If we consider the coordinates $\xi_{\mu}, \eta_{\mu}, \zeta_{\mu},(\mu=1,2,3)$, then the operators $\mathbf{N}$ take place of the operators $\mathbf{M}$ and vice versa. The relations

$$
\mathbf{M} \varphi(\xi, \eta, \zeta)=-(\mathbf{r} \times \nabla) \varphi(\xi, \eta, \zeta)
$$

exhibits this exchange. Thus, after the mappings in question we see instead of the operators of the angular momentum of the rotational motion the operators of the orbital angular momentum of the point particle. It is interesting that the relation

$$
\frac{1}{2} D \varphi(x, y, z)=x \frac{\partial \varphi}{\partial x}+y \frac{\partial \varphi}{\partial y}+z \frac{\partial \varphi}{\partial z} .
$$

holds valid in all instances.

Let us pay attention to the following important things. The coordinates of the four-dimensional Euclidian space are not observable but the picture of the rotation is very detailed and beautiful in this case and can be represented in the descriptive-geometric form. The coordinates of the threedimensional Euclidian space are quadratic functions of the coordinates of $R^{4}$ and are observable but the harmonic picture of rotation is reduced to the operators of the orbital angular momentum of the point particle. There is an interesting problem of half-integer orbital angular momentum which is in the sphere of interests of physicists up to now [2]. From our consideration it follows that eigenfunctions of the operators of angular momentum of the rotational motion can be the eigenfunctions of the operator of the orbital angular momentum only in the case when these functions are even, and this is the hidden reason of the integer eigenvalues.

\section{Conclusion}

In conclusion we give the answer on the following questions. What are the new results in your article? In what way are these new results timely? Why are these new results significant? 1.A new 
definition of space-time is given. The origin and nature of the rotational motion are recognized. It is established that on the four-dimensional Euclidian space there are two space-time structures and one of them is tightly connected with the rotational motion and a simply transitive group of turn dilatations. On this ground, the new basic equations for description of the so called strong interactions are suggested. 2. At the present time, quantum chromodynamics has no alternative, but in the framework of this theory we have no answer to the set of principle questions and hence new approaches are desirable. From this point of view our suggestion consider the leptons on the ground of one causal structure and connect the quarks with the other causal structure on the same four-dimension physical space looks like quite timely. 3. The problem of time and everything connected with this topic are always significant. The results obtained are significant because they give a simple and evident explanation of quark-lepton symmetry, quark confinement and baryon number conservation. From the point of view in question the baryon number conservation means that quarks cannot change the causal structure in which they live.

\section{References}

[1] I.B.Pestov, Particles \& Nuclei VOL. 44,PART 3, (2013), p.863.

[2] L.C.Biedenharn and J.D.Louck, Angular Momentum in Quantum Physics, Addison-Wesley Publishing Company, Inc, 1981.

[3] E.Cartan ,The Theory of Spinor, MIT Press, Cambridge, Mass., 1966.

[4] Shoshichi Kobayashi and Katsumi Nomizu, Foundations of Differential Geometry, Volume II, Intersience Publishers, New York-London-Sidney, 1969. 